\title{
The association of depression status with menopause symptoms among rural midlife women in China.
}

\author{
Hongyan Zang ${ }^{1}$, Lianping $\mathrm{He}^{2}$, Yan Chen², Jianfeng $\mathrm{Ge}^{2}$, Yingshui $\mathrm{YaO}^{2}$
}

1. Hongyan Zang, Women's health department, Yancheng Maternal and Child Health Hospital, Yancheng 224400, Jiangsu, People's Republic of China.

2. Lianping He , School of Public Health. Wannan Medical College. Wuhu 241002, Anhui,

People's Republic of China.

\begin{abstract}
Objective: This study aims to evaluate the association of depression with menopausal status and some menopause symptoms (vasomotor symptoms and poor sleep).

Methods: A total of 743 participants aged 40-60 years were recruited. Depression status was evaluated by using Self-Rating Depression Scale (SDS). Sleep quality and vasomotor symptoms were evaluated by specific symptoms questionnaire.

Results: The prevalence of depression among participants was $11.4 \%$. Depression was found more likely to occur in participants with poor sleep (OR, 6.02; 95\%CI, 3.61, 10.03) or with vasomotor symptoms (VMS) (OR, 2.03; 95\%CI, 1.20, 3.44) after controlling for age, education level, marital status, menopause status, monthly family income and chronic diseases. Menopause status was not associated with depression. Stratification analysis showed a significant association between poor sleep and depression across different menopause stages, while VMS were associated with depression only in premenopausal status.

Conclusion: The majority of Chinese rural midlife women do not experience depression. The relationship between depression, VMS and sleep disturbances tends to change with menopausal status in Chinese rural midlife women.
\end{abstract}

Keywords: depression, poor sleep, vasomotor symptoms, menopause, rural women

DOI: http://dx.doi.org/10.4314/ahs.v16i1.13

Cite as: Zang H, He L, Chen Y, Ge J, Yao Y. The association of depression status with menopause symptoms among rural midlife women in China. Afri Health Sci. 2016;16(1): 97-104. http://dx.doi.org/10.4314/abs.v16i1.13

\section{Introduction}

Depression is a common and major complaint among some mid-life women ${ }^{1}$. It adversely affects an individual's social relationship, capacity to work and learn, and is an indicator of the risk of self-harm and suicide ${ }^{2}$. Depression is also potentially harmful to physical health in addition to negatively impacting on women's psychosocial wellbeing ${ }^{2,3}$.

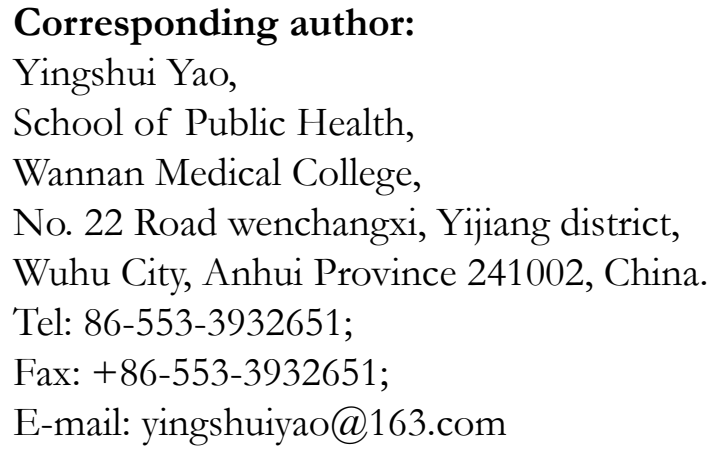

Some observational studies have suggested that the transition to menopause is a period of increased risk of depression in women and is linked to increased susceptibility to depression ${ }^{4,5}$. However, other studies reported that depression in midlife women may be more strongly associated with common menopausal symptoms (e.g. VMS and poor sleep) than menopause status per $\mathrm{se}^{6,7}$. There is no definitive consensus on whether depression is associated with menopausal symptoms and the menopause status. Moreover, most studies reporting association of depression with menopause status and menopause symptoms have been conducted in Western developed countries whereas the situation remains unclear in non-white populations including Asian women. Only recently, a population-based study found lower prevalence of depression in Taiwanese women than previous studies and suggested the disparity might be explained by racial and cultural differences ${ }^{8}$. Data from the Study of Women's Health across the Nation (SWAN) have also showed that the risk of depression is higher in African Americans but lower in Asian populations? 
The purpose of the current study was to evaluate the prevalence of depression in Chinese rural midlife women and explore the association of depression with menopausal status and menopause symptoms (VMS and poor sleep).

\section{Methods \\ Participants}

Data was obtained from a cross-sectional, communitybased study - the Yancheng Rural Midlife-Women Health Investigation. All participants were from three villages (Zhenghong, Caiqiao and Datao) of Yancheng prefecture in Jiangsu Province, China. Participants were randomly selected from a list of midlife women aged 40 to 60 years who received routine physical examination in community health service stations. Most of them were engaged in farming activities or small businesses and their living standards and lifestyles were typical of the majority of women in rural areas of Yancheng. Women who had received bilateral oophorectomy, pregnant and lactating women, and women on hormone drugs or other medications to alleviate symptoms associated with menopause were not included in the study. In total, 743 women out of a sample of 800 responded to our survey (response rate $92.9 \%$ ). The study was approved by the local Ethics Committee.

Face-to-face interviews were conducted by 8 trained interviewers and the investigators had the opportunity to explain the aims and conditions to the participants. Information was collected regarding age, education, marital status, menstrual history, average monthly family income and medical history etc.

\section{Measures}

The participants indicated whether they were experiencing VMS by selecting questionnaire items "I have hot flushes" or "I have night sweats" or "I have both".

Depression status was evaluated using the Self-rating Depression Scale (SDS) (Zung method) ${ }^{10}$. The SDS has been widely used to evaluate depression symptoms among adults. It is composed of 20 items describing depression symptoms. The Zung scale has been widely adopted to measure depression among Chinese women and also been adapted and tested in rural Chinese contexts with adequate reliability and validity and psychometric properties $^{11}$. According to Chinese norm, the total score of SDS would fall in a range from 4 to 80 points. Scores above 60 were labeled as having moderate or severe depression. Scores between 50 and 59 indicated having mild depression and scores under 50 points no depression. In the present study, a cut-off score was set at 50 .

Menopausal status was assessed by the following criteria: (1) women who had regular menstrual cycles (defined as cycles lasting from 23 to 35 days, or varying by $\leq 5$ days) over three months prior to the study were considered premenopausal; (2) women with irregular menstrual cycles (defined as cycles lasting $<23$ days, $>35$ days, or varying by $>5$ days) or women that ceased menstruation less than 12 months before were considered peri-menopausal; (3) absence of menstruation for more than 12 months was considered postmenopausal. Hormone levels were not obtained in all cases.

Marital status was coded as being married (including remarried and committed relationships) versus being single (including single, separated, divorced or widowed women).Sleep status was rated by responding to questionnaire items "How well do you sleep?" to which the alternative answer was either "poor" or "no problem". Response to "Chronic diseases" was either "yes" or "no", based on the presence or absence of a clinical diagnosis in the participants' health records of hypertension, heart diseases, diabetes, lung diseases and etc. Education status was assessed according to the number of years of schooling received.

\section{Statistical analysis}

The prevalence of current depression, poor sleep, VMS, and demographic/clinical characteristics (age, education, married status, monthly family income, chronic diseases) were presented as absolute and relative frequencies. Associations were analyzed using chi square tests.

Univariate logistic regression analysis was employed to determine whether VMS, poor sleep, menopause status and other characteristics were associated with the depression status. Multivariate logistic regression analysis was used to control for age, education, marital status, monthly family income, chronic diseases, and to examine whether poor sleep, VMS or menopause status was independently 
associated with depression status. Stratified analyses were performed according to menopausal status, and the relationship among depression, VMS, and poor sleep in each menopause group was examined separately.

Univariate and multivariate linear regression analyses were run with Zung as a numerical independent variable. Data was recorded with the EpiData software (version 3.0) and analyzed using the software SPSS 18.0. Adjusted odds ratios (OR) and their 95\% confidence intervals (CI) were calculated. A value of $\mathrm{p}<0.05$ was considered statistically significant.

\section{Results}

A total of 743 participants (334 pre-, 125 peri- and 284 postmenopausal women) were eventually assessed and $11.4 \%$ had depression. Characteristics of the participants by menopausal status are shown in Table 1. Most women were married and of low educational achievement but only a small percentage of them experienced depression, VMS, and poor sleep. Statistically significant differences were observed in the prevalence of chronic diseases, VMS, poor sleep, and depression among different menopause stages (pre-, peri-, and postmenopausal).

Table I. Characteristics of the participants by menopausal status

\begin{tabular}{|c|c|c|c|c|c|c|c|c|}
\hline \multirow{2}{*}{ Variables } & \multicolumn{2}{|c|}{ premenopausal } & \multicolumn{2}{|c|}{ Perimenopausal } & \multicolumn{2}{|c|}{ postmenopausal } & \multirow{2}{*}{ Total } & \multirow{2}{*}{$P$} \\
\hline & $\mathrm{n}$ & $\%$ & $\mathrm{n}$ & $\%$ & $\mathrm{n}$ & $\%$ & & \\
\hline Age(years) & & & & & & & & 0.000 \\
\hline $40-45$ & 210 & 62.87 & 49 & 39.20 & 6 & 2.11 & 265 & \\
\hline $46-50$ & 96 & 28.74 & 47 & 37.60 & 22 & 7.75 & 165 & \\
\hline $51-55$ & 22 & 6.59 & 27 & 21.60 & 140 & 49.30 & 189 & \\
\hline $56-60$ & 6 & 1.80 & 2 & 1.60 & 116 & 40.85 & 124 & \\
\hline Education(years) & & & & & & & & 0.000 \\
\hline$\leq 6$ & 182 & 54.49 & 75 & 60.00 & 212 & 74.65 & 469 & \\
\hline $6-9$ & 122 & 36.53 & 39 & 31.20 & 45 & 15.85 & 206 & \\
\hline$>9$ & 30 & 8.98 & 11 & 8.80 & 27 & 9.51 & 68 & \\
\hline Married status & & & & & & & & 0.439 \\
\hline Being married ${ }^{a}$ & 326 & 97.60 & 121 & 96.80 & 272 & 95.77 & 719 & \\
\hline Being single & 8 & 2.40 & 4 & 3.20 & 12 & 4.23 & 24 & \\
\hline \multicolumn{3}{|l|}{ Monthly family income (yuan) } & & & & & & 0.000 \\
\hline $0-1000$ & 65 & 19.46 & 37 & 29.60 & 114 & 40.14 & 216 & \\
\hline $1000-3000$ & 234 & 70.06 & 77 & 61.60 & 157 & 55.28 & 468 & \\
\hline$>3000$ & 35 & 10.48 & 11 & 8.80 & 13 & 4.58 & 59 & \\
\hline Chronic diseases & & & & & & & & 0.000 \\
\hline No & 237 & 70.96 & 79 & 63.20 & 154 & 54.23 & 470 & \\
\hline Yes & 97 & 29.04 & 46 & 36.80 & 130 & 45.77 & 273 & \\
\hline Sleep & & & & & & & & 0.011 \\
\hline No problem & 277 & 82.93 & 101 & 80.80 & 208 & 73.24 & 586 & \\
\hline Poor sleep & 57 & 17.07 & 24 & 19.20 & 76 & 26.76 & 157 & \\
\hline VMS & & & & & & & & 0.000 \\
\hline No & 284 & 85.03 & 92 & 73.60 & 199 & 70.07 & 575 & \\
\hline Yes & 50 & 14.97 & 33 & 26.40 & 85 & 29.93 & 168 & \\
\hline Depression & & & & & & & & 0.000 \\
\hline Nondepressed & 311 & 93.11 & 113 & 90.40 & 235 & 82.75 & 659 & \\
\hline Depressed & 23 & 6.89 & 12 & 9.60 & 49 & 17.25 & 84 & \\
\hline
\end{tabular}


Table II shows that midlife women with depression were more likely to experience poor sleep and VMS. Age, family income, and menopause status were also found to be significantly associated with depression but education level and the state of being married were not. After adjusting for age, education level, marital status, chronic disease, and family income, we found that depression was significantly associated with poor sleep and VMS but not with menopausal status.

Table II The association of depression, menopause status and menopause symptoms

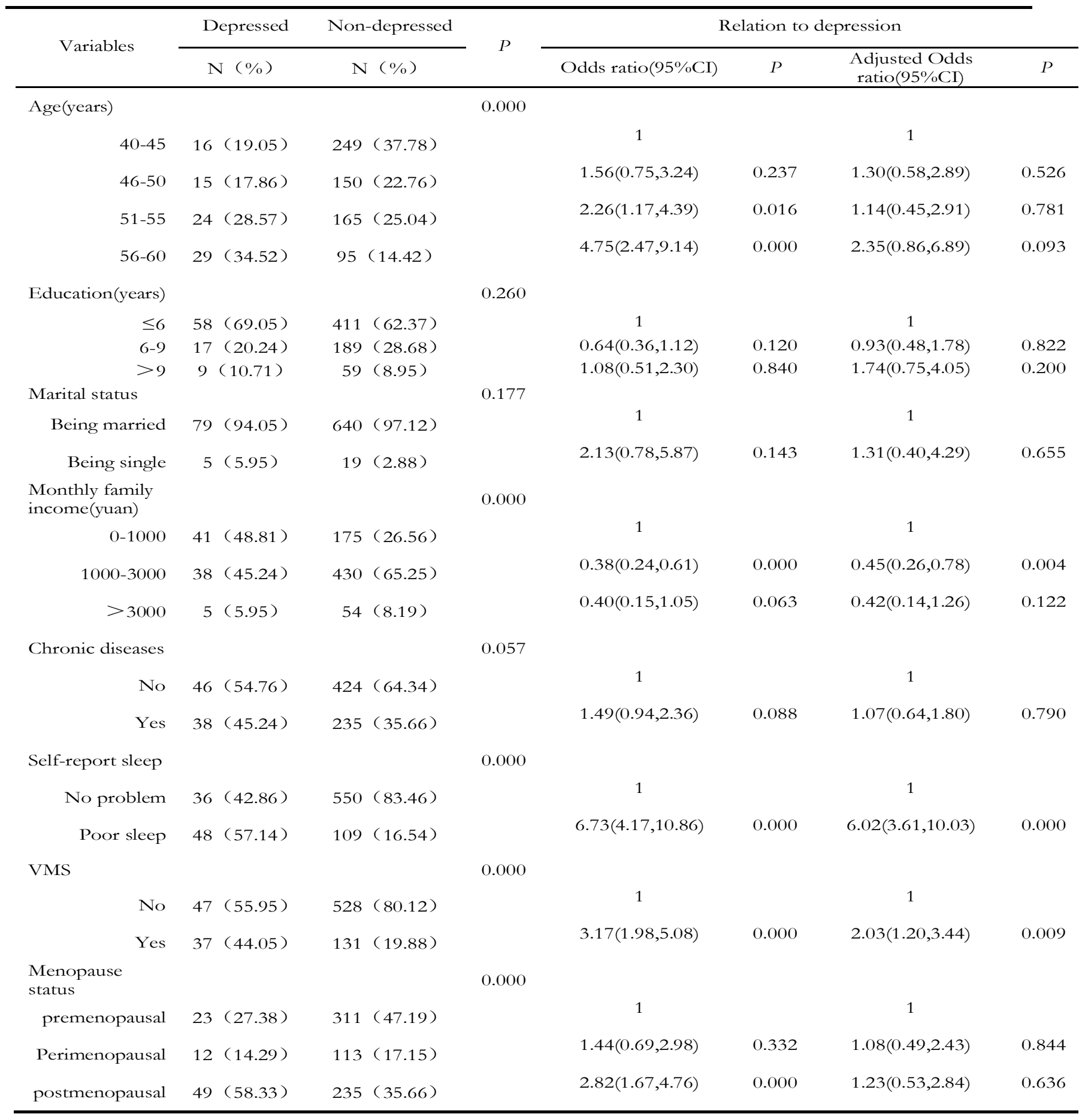


VMS and sleep problems, independent of each other, were significantly associated with depression during the pre-menopausal period. But in the peri- and post-meno- pausal periods, only poor sleep alone was associated with depression when several other confounders were controlled for (Table III).

Table III. The relationship between depression, VMS and poor sleep by menopause status according to stratify analyses

\begin{tabular}{|c|c|c|c|c|c|c|c|c|c|c|c|c|}
\hline & \multicolumn{12}{|c|}{ Menopause status } \\
\hline & \multicolumn{4}{|c|}{ premenopause } & \multicolumn{4}{|c|}{ perimenopause } & \multicolumn{4}{|c|}{ postmenopause } \\
\hline & $\begin{array}{c}\text { Odds ratios }^{\text {a }} \\
(95 \% \mathrm{CI})\end{array}$ & $P$ & $\begin{array}{c}\text { Odds ratios } \\
(95 \% \mathrm{CI})\end{array}$ & $P$ & $\begin{array}{c}\text { Odds ratios }^{\mathrm{a}} \\
(95 \% \mathrm{CI})\end{array}$ & $P$ & $\begin{array}{c}\text { Odds ratios } \\
(95 \% \mathrm{CI})\end{array}$ & $P$ & $\begin{array}{c}\text { Odds ratios }^{\mathrm{a}} \\
(95 \% \mathrm{CI})\end{array}$ & $P$ & $\begin{array}{c}\text { Odds ratios } \\
(95 \% \mathrm{CI})\end{array}$ & $P$ \\
\hline VMS & $2.87(1.12,7.37)$ & 0.029 & $2.90(1.04,8.07)$ & 0.041 & $2.29(0.65,8.05)$ & 0.195 & $1.72(0.41,7.21)$ & 0.461 & $1.63(0.80,3.33)$ & 0.178 & $1.69(0.80,3.55)$ & 0.167 \\
\hline $\begin{array}{l}\text { Poor- } \\
\text { sleep }\end{array}$ & $3.02(1.21,7.54)$ & 0.018 & $3.23(1.23,8.47)$ & 0.017 & $3.68(1.04,13.05)$ & 0.044 & $4.19(1.06,16.51)$ & 0.041 & $9.25(4.54,18.85)$ & 0.000 & $11.5(5.39,24.66)$ & 0.000 \\
\hline
\end{tabular}

\section{Discussion}

A small fraction of participants (11.4\%) of this study was found to have experienced depression lower than reported in Europe and the United States ${ }^{4,7,9}$ and higher than found in Chinese women $(2.76 \%-4.56 \%)$ studied in Beijing ${ }^{12}$. The gaps between these study results might be attributed to the different characters of the populations sampled (such as culture, lifestyle, marital disruption and nutrition/diet habits) and to the different measure tools employed ${ }^{4,9}$. Our participants were from rural areas, aged 40 to 60 years. They experienced poverty and food shortages during their childhood years, but led relatively wealthier lives now thanks to rapid development of economy in recent decades. Most of them were engaged in farming activities or small businesses. Divorce rate was very low among these rural women who were under very strong influence of traditional family values. Prominent characters of our participants such as living standard and lifestyle, typical of the majority of Yancheng rural women, might contribute to the relatively lower prevalence of depression among them.

Furthermore, depression was found significantly associated with VMS and poor sleep, but not with menopausal status. Poor sleep was independently associated with increased risk of depression across the menopausal status range. However, VMS were only associated with increased risk of depression during the pre-menopausal, but not the peri- and postmenopausal periods. To avoid spurious statistical significance due to the use of depression as a dichotomous variable with a SDS cutoff score of 50, we ran additional univariate and multivariate linear regression analyses treating depression as an independent numerical variable. The results were comparable to those from the logistic regression analysis.

The current study showed that only the post menopausal period was associated with depression but the relationship changed after controlling for several confounders. The result is consistent with a previous study conducted on Taiwanese rural population-based people ${ }^{13,14}$. Some studies suggest that menopausal status is linked to increased risk of depression mainly because of hormonal flux ${ }^{5,15-18}$. There are several explanations for the discrepancy among these results. First, findings mainly negating the connection between menopause status and depression were made in Asian rural midlife women (ours included) and indicated that the relationship might also be subject to the influence of ethnicity and other socio-demographic factors. Second, depression in different studies was measured using different tools $\mathrm{s}^{4,14}$.

In the current study, the women who reported poor sleep were more than six times as likely to have depression as 
those without sleep problems (OR, 6.02; 95\%CI, 3.61, 10.03) and stratification analyses supported the significant association across three menopausal stages. Our study suggests that sleep problems and depression are strongly connected throughout midlife, corroborating the views in previous studies ${ }^{19,20}$. There has been literature suggesting that poor sleep is a major manifestation or a prodromal symptom of depression which may be a predominant cause for visiting a doctor ${ }^{4,21-23}$. It is thus important for midlife women to receive treatment for sleep problems regardless of menopausal status, in order to prevent or relieve possible accompanying depression symptoms.

As expected, peri- and postmenopausal women reported more VMS than pre-menopausal women. However, VMS were found associated with depression only in the premenopausal period (not in the peri- and postmenopausal periods) in our study. Our results vary from previous studies which reported association of depression with VMS in the peri- and postmenopausal periods or throughout three menopausal stages ${ }^{2,6,7}$. One plausible explanation for the differences between these studies is that premenopausal women with VMS may be under more stress than peri- and postmenopausal women. Premenopausal women would feel despondent or stressful when they experience VMS which never or seldom occurred to them before while peri- and postmenopausal women might have already got accustomed to VMS. Another plausible explanation may be the difference in terms of age, education level, income, and chronic diseases between the three stages.

The current study surveyed a relatively large sample from an eastern Asian rural population previously under represented in the literature but assumed to have low frequency of depression, VMS, and sleep problems. Several demographical, clinical confounders and different menopausal status were assessed, which were the strengths of this study. The principle finding is that the prevalence of depression did not vary with women's menopausal status. However, association between VMS and depression was only observed in the premenopausal women. This is in line with previous data from rural Taiwanese population displaying low frequency of VMS, depression, and poor sleep. Common menopausal symptoms (VMS) and menopause status might not be major contributors to depression among midlife women with lower prevalence of
VMS and depression. Further work is needed to investigate the cause of low level depression in midlife women with low level VMS and sleep problems. A longitudinal study design may be conducive to elucidating the effects of VMS and stressful life events on depression, particularly in the premenopausal women.

\section{Limitations}

Some limitations of the study should be taken into account. First, this was a cross-sectional survey and we could not clarify the causality of association. Secondly, sleep disturbance and VMS were assessed by self-reported questionnaires rather than detailed and objective measurements and possible reporting bias should be considered. Thirdly, the Zung scale alone was not an adequate procedure for diagnosing depression. A clear diagnosis should have ideally been obtained by a clinical psychiatrist on the basis of professional judgment and using structured assessment tools. Then, age and menopause are highly correlated and may confound each other. It is difficult to adequately adjust for age despite the fact that we controlled for it in the regression analysis. Next, some important information such as BMI recordings, history of depression, use of psychoactive drugs and drinking habit of tea that could affect sleep and mood were not collected. Finally, menopausal status was assessed by menstrual history rather than by testing hormonal levels.

\section{Conclusion}

Our results provide a new perspective on the association between depression with menopausal status and some menopausal symptoms (VMS and poor sleep). Menopausal status may not be associated with depression. There is a significant association between poor sleep and depression across all three menopause status, whereas the association between VMS and depression is significant only in the premenopausal women. Health professionals caring for menopausal women should be particularly careful in assessing sleep patterns throughout menopausal transition. Assessment of VMS may be of more importance during the perimenopausal period for prevention or monitoring of depression.

\section{Acknowledgements}

This research was funded by Yancheng Medical Science and Technology Development Program (YK2011027)

The authors thank Prof. Xiao Chen for his assistance in language editing of this paper. 


\section{Disclosure of conflict of interest}

None

\section{References}

1. Bromberger JT, Kravitz HM, Chang YF, Cyranowski JM, Brown C, Matthews KA. Major depression during and after the menopausal transition: Study of Women's Health Across the Nation (SWAN). Psychol Med. 2011. 41(9): 1879 -88. PubMed. Doi:10.1017/S003329171100016X.

2. Clayton A, Guico-Pabia C. Recognition of depression among women presenting with menopausal symptoms. Menopause. 2008. 15: 758-67. (4 PubMed Pt 1)

3. Perez-Lopez FR, Chedraui P, Gilbert JJ, Perez-Roncero G. Cardiovascular risk in menopausal women and prevalent related co-morbid conditions: facing the post-Women's Health Initiative era. Fertil Steril. 2009. 92(4): 1171 -86. PubMed. Doi: 10.1016/j.fertnstert.2009.06.032.

4. Cohen LS, Soares CN, Vitonis AF, Otto MW, Harlow BL. Risk for new onset of depression during the menopausal transition: the Harvard study of moods and cycles. Arch Gen Psychiatry. 2006. 63(4): 385-90. Doi:10.1001/ archpsyc.63.4.385.

5. Freeman EW, Sammel MD, Lin H, Nelson DB. Associations of hormones and menopausal status with depressed mood in women with no history of depression. Arch Gen Psychiatry. 2006. 63(4): 375-82. Doi: 10.1001/ archpsyc.63.4.375.

6. Gallicchio L, Schilling C, Miller SR, Zacur H, Flaws JA. Correlates of depressive symptoms among women undergoing the menopausal transition. I Psychosom Res. 2007. 63(3): 263 -8. PubMed Doi: 10.1016/j.jpsychores.2007.02.003.

7. Bosworth HB, Bastian LA, Kuchibhatla MN, et al. Depressive symptoms, menopausal status, and climacteric symptoms in women at midlife. Psychosom Med. 2001. 63(4): 603 -8. PubMed

8. Lin HL, Hsiao MC, Liu YT, Chang CM. Perimenopause and incidence of depression in midlife women: a population-based study in Taiwan. Climacteric. 2013. 16(3): 381 -6. PubMed. Doi: 10.3109/13697137.2012.707706.

9. BrombergerJT, Matthews KA, Schott LL, et al. Depressive symptoms during the menopausal transition: the Study of Women's Health Across the Nation (SWAN). J Affect Disord. 2007. 103:267-72. (1-3 PubMed ). Doi: 10.1016/j.jad.2007.01.034.

10. Zung WW, Richards CB, Short MJ. Self-rating depression scale in an outpatient clinic. Further validation of the SDS. Arch Gen Psychiatry. 1965. 13(6): 508 -15. PubMed.
11. Peng H, Zhang YY, Ji Y, Tang WQ, Li Q, Yan XL, et al. Analysis of reliability and validity of Chinese version of SDS scale in women of rural area. Shanghai Medical and Pharmacentical Journal (14), 2013. 20-22,23. Doi: 10.3969/j. issn.1006-1533.2013.14 .011. PubMed.

12. Zhang SW, Wang JH, Zhou H, Rao KQ, Deng XH. Survey of depressive symptoms among menopausal women in Beijing. Chinese Mental Health Journal 2003;17:34850,347. Doi:10.3321/j.issn:1000-6729.2003.05 .024. PubMed.

13. Juang KD, Wang SJ, Lu SR, Lee SJ, Fuh JL. Hot flashes are associated with psychological symptoms of anxiety and depression in peri- and post- but not premenopausal women. Maturitas. 2005. 52(2): 119 -26. PubMed. Doi: 10.1016/j.maturitas.2005.01.005.

14. Shaver JL. The interface of depression, sleep, and vasomotor symptoms. Menopause. 2009. 16(4): 626-9. Doi: 10.1097/gme.0b013e3181a9c54f.

15. Freeman EW, Sammel MD, Liu L, Gracia CR, Nelson DB, Hollander L. Hormones and menopausal status as predictors of depression in women in transition to menopause. Arch Gen Psychiatry. 2004. 61(1): 62 PubMed -70. Doi: 10.1001/archpsyc.61.1.62

16. Joffe H, Hall JE, Soares CN, et al. Vasomotor symptoms are associated with depression in perimenopausal women seeking primary care. Menopause. 2002. 9(6): 392 -8. PubMed

17. Schmidt PJ, Haq N, Rubinow DR. A longitudinal evaluation of the relationship between reproductive status and mood in perimenopausal women. Am J Psychiatry. 2004. 161(12): 2238 PubMed -44. Doi: 10.1176/appi. ajp.161.12.2238.

18. Soares CN. Can depression be a menopause-associated risk. BMC Med. 2010. 8:79. PubMed. Doi: 10.1186/17417015-8-79.

19. Franzen PL, Buysse DJ. Sleep disturbances and depression: risk relationships for subsequent depression and therapeutic implications. Dialogues Clin Neurosci. 2008. 10(4): 473-81.

20. Polo-Kantola P, Laine A, Aromaa M, et al. A population-based survey of sleep disturbances in middle-aged women--associations with health, health related quality of life and health behavior. Maturitas. 2014. 77(3): 255 -62. PubMed. Doi: 10.1016/j.maturitas.2013.11.008.

21. Brown JP, Gallicchio L, Flaws JA, Tracy JK. Relations among menopausal symptoms, sleep disturbance and depressive symptoms in midlife. Maturitas. 2009. 62(2): 184 -9. PubMed. Doi: 10.1016/j.maturitas.2008.11.019. 
22. Terauchi M, Hiramitsu S, Akiyoshi M, et al. Associations between anxiety, depression and insomnia in periand post-menopausal women. Maturitas. 2012. 72(1): 615. PubMed. Doi: 10.1016/j.maturitas.2012.01.014.
23. Kloss JD, Tweedy K, Gilrain K. Psychological factors associated with sleep disturbance among perimenopausal women. Behav Sleep Med. 2004. 2(4): 177-90. PubMed. Doi: 10.1207/s15402010bsm0204_1. 